\title{
DINÁMICA DE LA CONCENTRACIÓN DE COBRE, PLOMO, MERCURIO Y ARSÉNICO EN SEDIMENTOS DEL RÍO COPIAPÓ, CHILE
}

\author{
(Dynamics of copper, lead, mercury and arsenic concentration in sediments of the Copiapó river, Chile)
}

\author{
Seabastián ROJOS ${ }^{1}$, Germán AGUILAR $^{2}$, Bernardo SEPÚLVEDA ${ }^{1 *}$ y Osvaldo PAVEZ ${ }^{1,3}$
}

${ }^{1}$ Centro Regional de Investigación para el Desarrollo Sustentable de Atacama (CRIDESAT), Universidad de Atacama, Copayapu 485, Región de Atacama, Copiapó, Chile

${ }^{2}$ Centro Avanzado de Tecnología para la Minería, Facultad de Ciencias Físicas y Matemáticas, Universidad de Chile, Tupper 2007, Santiago, Chile

${ }^{3}$ Departamento de Ingeniería en Metalurgia, Universidad de Atacama, Chile

*Autor para correspondencia: bernardo.sepulveda@uda.cl

(Recibido noviembre 2017; aceptado julio 2018)

Palabras clave: transferencia de elementos, pasivos ambientales mineros, desierto de Atacama

\section{RESUMEN}

La cuenca del río Copiapó, al sur del desierto de Atacama, tiene una fuerte intervención antrópica debido a la actividad minera, agrícola y urbana. La presencia de pasivos ambientales mineros en la cuenca es relevante, ya que éstos se encuentran cerca de cursos de agua y zonas pobladas. En este trabajo se estudió la relación entre la variación de concentraciones de cobre, plomo, mercurio y arsénico con la dinámica de trasporte de sedimentos a lo largo de un año. Se analizó la granulometría y composición química de los sedimentos presentes en cinco puntos del río Copiapó, incluyendo zonas de intervención minera, agrícola y urbana. Los resultados muestran que la variación temporal de la concentración de los elementos analizados es inversamente proporcional al incremento de la transferencia de la fracción más fina de sedimento. Lo anterior se relaciona con cambios en el transporte de sedimentos, el cual está influido por lluvias esporádicas, deshielo, aporte de sedimentos desde aguas arriba y eventos eólicos en las zonas con actividad minera.

Key words: transfer of elements, mining environmental tailings, Atacama desert

\begin{abstract}
The Copiapó river basin, to the south of the Atacama desert, has a strong anthropic intervention due to mining, agricultural and urban activities. The presence of mining environmental tailings in the basin is relevant, mainly when they are close to watercourses and populated areas. In this work the relationship between the variations of copper, lead, mercury and arsenic concentrations related to the sediment transport dynamics along a year was studied. From five points along the Copiapó river, including areas of mining, agricultural and urban intervention, the sediments were analyzed for granulometry and chemical composition. The results show that the temporal variation
\end{abstract}


of elements concentrations was proportionally inverse to the transport of the finest fraction of the sediment. This is related to changes in sediments transport, influenced by sporadic rains, snow melting, sediment supply from upstream sediments and wind events in areas with mining activity.

\section{INTRODUCCIÓN}

La cuenca del río Copiapó ha estado sometida a una fuerte intervención antrópica debida a la actividad minera, agrícola y urbana desarrollada desde finales del siglo XIX (Castro et al. 2009, Espinoza 2013). La presencia de pasivos ambientales mineros adquiere especial relevancia al localizarse cerca de cauces fluviales y zonas pobladas. Entre los pasivos ambientales se reconocen relaves, residuos metalúrgicos de procesos de flotación de plantas concentradoras de $\mathrm{Cu}$, depósitos de escorias de $\mathrm{Cu}$ y relaves de pequeña minería que usa $\mathrm{Hg}$. Se han identificado plantas abandonadas de cianuración y amalagamación de $\mathrm{Ag}$ en las localidades de $\mathrm{Pa}$ bellón, Totoralillo y Potrero Seco, con niveles de $\mathrm{Hg}$ de hasta $1800 \mathrm{~g} / \mathrm{t}$, recomendándose determinar el peligro potencial y poner en práctica medidas de seguridad (Eberle 1998). Todos estos residuos son fuentes potenciales de metales que se pueden traducir en riesgos de salud para la población (Jara 2007). La problemática descrita ha estimulado a un grupo interdisciplinario de profesionales a realizar un monitoreo de las concentraciones de $\mathrm{Cu}, \mathrm{Pb}, \mathrm{Hg}$ y As en sedimentos fluviales en el valle del río Copiapó. El monitoreo incluyó áreas con fuerte presencia de pasivos ambientales mineros y otras relativamente libres de estos residuos.

En el presente trabajo se realizó un estudio geoquímico sedimentario en la cuenca de dicho río para conocer la relación entre la variación de concentraciones de $\mathrm{Cu}, \mathrm{Pb}, \mathrm{Hg}$ y As con la dinámica de trasporte de sedimentos a lo largo de un año, la cual puede estar influida por mecanismos bióticos y abióticos que modifican la dinámica de transferencia de materia en la cuenca (Bugueño 2014). El río Copiapó está sometido a un fuerte estrés hídrico, derivado de la explotación de aguas por la industria minera y agrícola. En este contexto, el segmento del río donde se concentra la industria y la población presenta escorrentía que se activa sólo durante periodos de lluvias ocasionales y extraordinarios periodos de deshielo en la cordillera de los Andes.

\section{MATERIALES Y MÉTODOS}

\section{Muestreo}

El muestreo de sedimentos del río Copiapó se hizo mensualmente durante el año hidrológico 2011-2012. Se seleccionaron cinco puntos de muestreo (Fig. 1) a lo largo del cauce del río Copiapó desde aguas arriba:

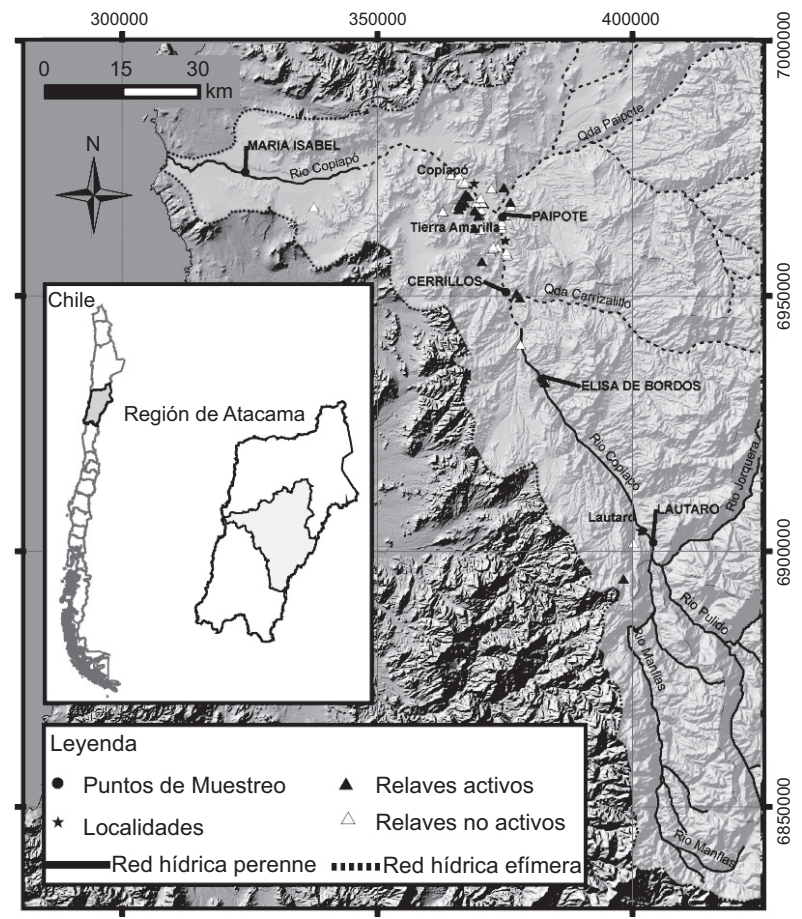

\begin{tabular}{|c|l|r|r|r|}
\hline$p$ & & \multicolumn{2}{|c|}{ Coordenadas UTM } & MSNM \\
\hline 1 & Embalse Lautaro & $403731.66 \mathrm{E}$ & $6902209.00 \mathrm{~S}$ & 2254 \\
\hline 2 & Elisa de Bordo & $382151.85 \mathrm{E}$ & $6933555.13 \mathrm{~S}$ & 1367 \\
\hline 3 & Puente Cerrillos & $375420.26 \mathrm{E}$ & $6950520.80 \mathrm{~S}$ & 960 \\
\hline 4 & Paipote & $374555.05 \mathrm{E}$ & $6965450.94 \mathrm{~S}$ & 447 \\
\hline 5 & María Isabel (DGA) & $317939.13 \mathrm{E}$ & $6976437.45 \mathrm{~S}$ & 142 \\
\hline
\end{tabular}

MSNM = metros sobre el nivel del mar, DGA = Punto de muestreo de la Dirección General de Aguas

Fig. 1. Ubicación de puntos de muestreo, situación hídrica (perenne y efímera), y localización de pasivos ambientales mineros en el curso del río Copiapó 
1) Tranque Lautaro (LAU), 2) Elisa de Bordos (ELI), 3) Puente Cerrillos (CER), 4) Paipote (PAI) y 5) Fundo María Isabel (ISA). Los puntos de muestreo del 1 al 3 están localizados en la subcuenca media y el resto en la subcuenca baja del río Copiapó. Los puntos 1,2 y 5 son de escorrentía perenne y el 3 y el 4 de escorrentía efímera. Cabe destacar que el punto de muestreo 5, si bien se localiza en un segmento de escorrentía perenne, está desconectado hídricamente del resto al ubicarse aguas abajo del segmento de escorrentía efímera. En la actualidad, entre los puntos 1 y 4 hay actividad agrícola, principalmente cultivo de vid y algunas hortalizas. Entre los puntos 2 y 4 hay actividad minera, mientras que en el punto 5 existe cultivo extensivo de olivo y no hay minería.

Las muestras fueron de aproximadamente $3 \mathrm{~kg}$ de sedimento depositado en los últimos $5 \mathrm{~cm}$ (superficial). La extracción se realizó con materiales plásticos para evitar contaminación por metales; se eliminó material superior a $12.7 \mathrm{~mm}$. Las muestras fueron envasadas en bolsas de polietileno y rotuladas según la posición y fecha de muestreo.

\section{Granulometría}

Las muestras se secaron a $30{ }^{\circ} \mathrm{C}$ en el Instituto de Investigaciones Científicas y Tecnológicas (IDICTEC) de la Universidad de Atacama, para evitar cambios en la mineralogía. Luego se caracterizaron granulométricamente, usando tamices (13) con aberturas de 6.25 a $0.075 \mathrm{~mm}$. Para caracterizar las fracciones de sedimentos transportados por tracción, saltación y suspensión, se obtuvo el porcentaje del peso de cada una y se realizaron curvas de frecuencia granulométricas acumulativas (Spalletti 1980). Se llevó a cabo un análisis estadístico mediante el método de los cuartiles, desde las curvas de frecuencia granulométricas acumulativas de ordenada aritmética según el método de Folk y Ward (1957) y de los datos obtenidos de la curva acumulativa de ordenada probabilística según Visher (1969). Asimismo, se aplicó el método de los momentos a partir de las frecuencias y del valor medio de cada intervalo granulométrico (Friedman y Sanders 1978).

Para cada punto de muestreo, se graficó el porcentaje en peso acumulado retenido de sedimento por estación vs. el tamaño de la partícula en $\Phi$, indicándose la fecha de muestreo de cada serie de datos. Desde cada gráfico se determinaron las secciones de la curva resultante con correlación de al menos $r=0.9$. Las rectas de tendencia se graficaron e igualmente las intersecciones a las inflexiones de la curva promedio de acumulación, en correspondencia. Cada intersección separa los distintos tipos de transporte (tracción, saltación, suspensión) y la pendiente de cada recta es indicativa del porcentaje en peso de cada población en la muestra.

Se calculó la significancia de las pendientes entre tipos de transporte mediante la prueba t de Student para muestras pareadas, obteniéndose un índice $\mathrm{p}$ de $5 \%$ de error $(\mathrm{p}=0.05)$ como límite para determinar la significancia.

\section{Caracterización química}

El proceso metalúrgico asociado a los relaves implica que éstos estén constituidos por material de granulometría aproximadamente inferior a 1 $\mathrm{mm}$. Para conocer la influencia de los relaves en los sedimentos fluviales, se determinó la concentración de $\mathrm{Cu}, \mathrm{Pb}, \mathrm{Hg}$ y As en la fracción de granulometría inferior a $0.075 \mathrm{~mm}$ de las muestras recolectadas mensualmente durante el periodo 2011-2012 en los cinco puntos de muestreo.

En el análisis de $\mathrm{Cu}, \mathrm{Pb}$ y As, muestras de $1 \mathrm{a} 2 \mathrm{~g}$ de sedimento se sometieron a digestión ácida en 25 $\mathrm{mL}$ de $\mathrm{HNO}_{3}$ a $80^{\circ} \mathrm{C}$ en plancha calefactora, hasta la reducción de volumen a $5 \mathrm{~mL}$. A la mezcla de digestión, una vez fría, se agregaron $5 \mathrm{~mL}$ de ácido perclórico. La mezcla se calentó en plancha calefactora a $200{ }^{\circ} \mathrm{C}$ hasta llegar al estado siruposo; luego se adicionaron $2 \mathrm{~mL}$ de ácido clorhídrico concentrado y se aforó a $100 \mathrm{~mL}$ con agua destilada.

La concentración de $\mathrm{Cu}$ y $\mathrm{Pb}$ fue determinada por espectrofotometría de absorción atómica con atomización a la llama y con aire de acetileno, para lo que se utilizó un espectrofotómetro de absorción atómica (GBC Avanta).

La concentración de As y $\mathrm{Hg}$ se determinó por generación de hidruros en el generador HG-3000 acoplado al espectrofotómetro de absorción atómica.

Para determinar el $\mathrm{Hg}$ se usó la técnica de vapor frío. La concentración de vapor de $\mathrm{Hg}$ se determinó en el analizador $\mathrm{Hg}$-Analuser $254 \mathrm{~N}$, previamente calibrado con patrones de concentración de 1, 5 y 10 ppb. Todos los reactivos utilizados fueron de grado proanálisis, no obstante en cada determinación se realizó un blanco de reactivos. Se estimó la variación mensual de concentración de cada elemento en cada punto de muestreo.

En el hemisferio sur las estaciones del año se establecen en los siguientes periodos: verano, del 21 de diciembre al 20 de marzo; otoño, del 21 de marzo al 20 de junio; invierno, del 21 de junio al 20 de septiembre, y primavera del 21 de septiembre al 20 de diciembre. Para cada elemento se obtuvo una línea base correspondiente al valor de concentración mensual ambientalmente permisible para ser 
considerado contaminante, según lo establecido en las Canadian Enviromental Quality Guidelines (CCME, 2018), ya que se trata de un estándar ambiental alto a nivel mundial.

\section{RESULTADOS}

\section{Granulometría y transporte}

Con los datos de la caracterización granulométrica por tamizado de sedimentos, se graficó el peso acumulado (\%) vs. el tamaño promedio de las partículas ( $\Phi$ en $\mathrm{mm}$ ) en escala logarítmica de base 2; según la ecuación $\Phi=-\log 2$ diámetro ( $\mathrm{mm})$, la escala de tamaño usada (Visher 1969) se representa en la figura 2. El tamaño granulométrico está comprendido entre $-3 \Phi$, correspondiente a grava fina de $8 \mathrm{~mm}$, hasta $5 \Phi$, correspondiente a limo grueso de $0.031 \mathrm{~mm}$.

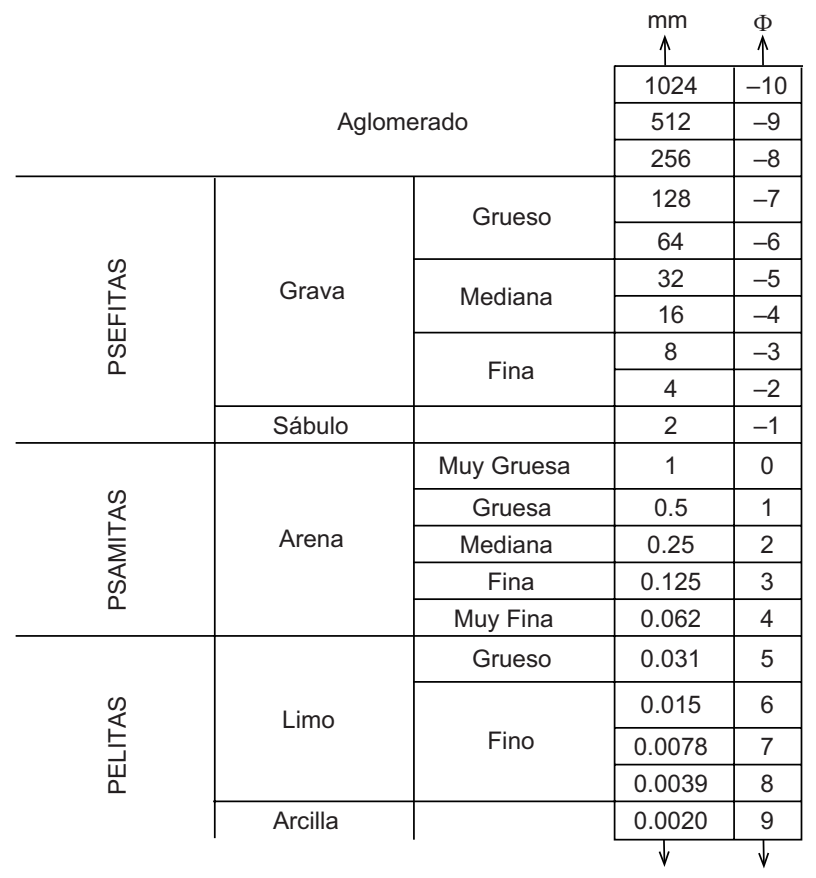

Fig. 2. Cuadro comparativo de la escala milimétrica y la escala granulométrica de valores phi $(\Phi)$ equivalentes

La figura 3 muestra la acumulación de sedimento en Tranque Lautaro, primer punto de muestreo. En el otoño no existió transporte por tracción ni saltación. En invierno se encontraron todos los tipos de transporte; las pendientes de tracción y saltación fueron significativamente diferentes $(p=0.006)$ y la pendiente de suspensión fue significativamente diferente a la de saltación $(p=0.03)$. En primavera no hubo saltación y una pequeña cantidad de sedimento se transportó por tracción. En verano se encontró gran cantidad de sedimento transportado por saltación y una pequeña cantidad por suspensión. Para cada muestra de sedimento entre 3 y $4 \Phi(0.125$ y 0.062 $\mathrm{mm})$ se realizó un análisis químico.

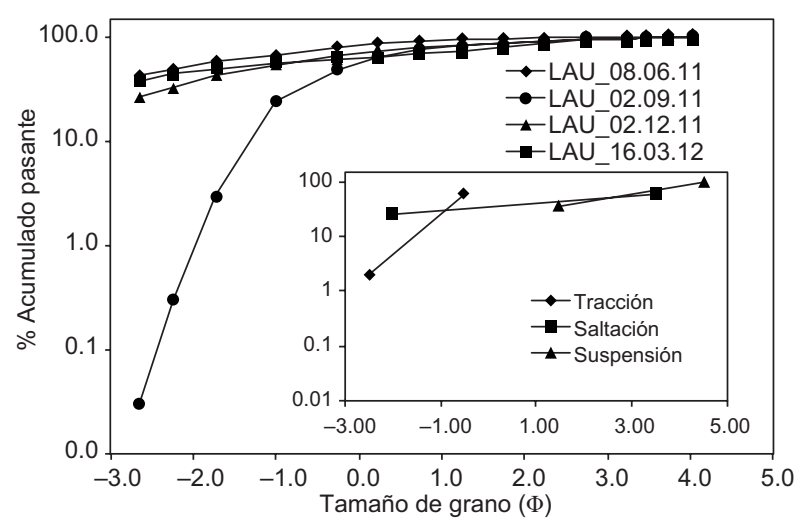

Fig. 3. Peso retenido acumulado en relación con el tamaño de partícula $(\Phi)$ de sedimentos en el punto de muestreo Tranque Lautaro (LAU) en cuatro fechas. En el recuadro interno del gráfico se muestran las líneas representativas de los tipos de transporte

En la figura 4 se presenta la acumulación de sedimento para el punto de muestreo Elisa de Bordos. En otoño, invierno y verano se presentaron los tres tipos de transporte (tracción, saltación y suspensión, $\mathrm{p}<0.05)$, mientras que en primavera disminuyó la tracción.

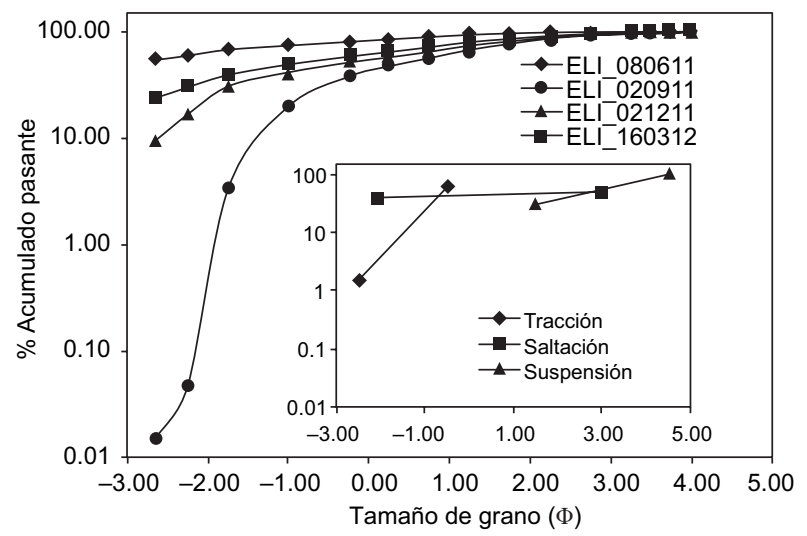

Fig. 4. Relación del peso retenido acumulado en relación con el tamaño de partícula $(\Phi)$ de sedimentos en el punto de muestreo Elisa de Bordos (ELI). En el recuadro interno del gráfico se muestran las líneas representativas de los tipos de transporte 
En la figura 5 se muestra la acumulación de sedimento para el punto de muestreo Puente Cerrillos. En otoño y primavera se presentó transporte de partículas por tracción y suspensión; en invierno y verano se presentaron los tres tipos de transporte $(\mathrm{p}<0.05)$.

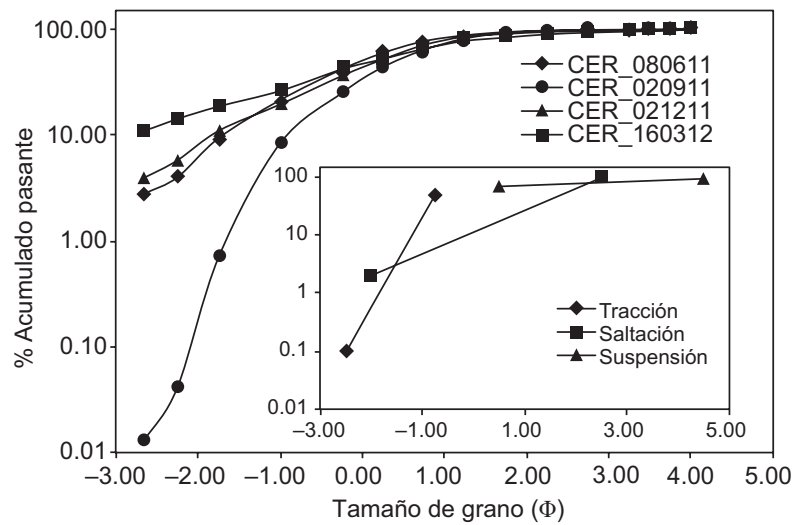

Fig. 5. El peso retenido acumulado con relación al tamaño de partícula $(\Phi)$ de sedimentos en el punto de muestreo Puente Cerrillos (CER). En el recuadro interno del gráfico se muestran las líneas representativas de los tipos de transporte

En la figura 6 se exhibe la acumulación de sedimento para el punto de muestreo Paipote. En otoño se regsitró transporte de partículas por tracción y saltación, en tanto que en invierno, primavera y verano ocurrió transporte por tracción y suspensión $(\mathrm{p}<0.05)$.

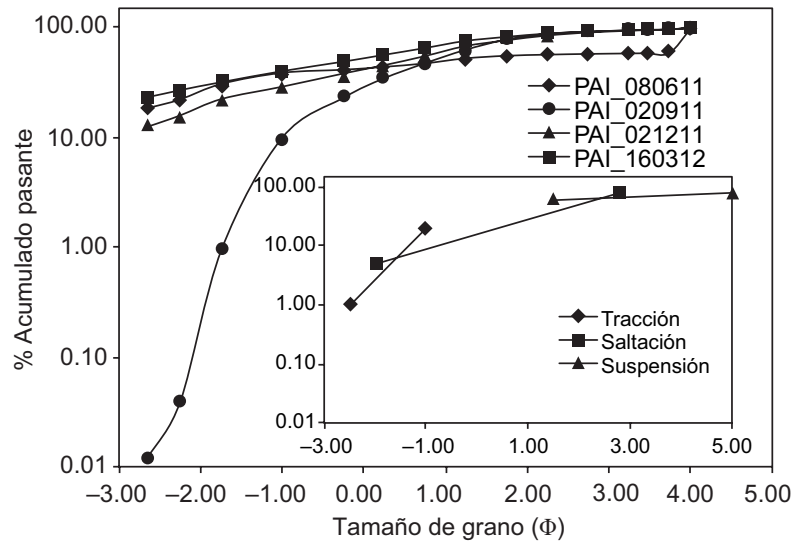

Fig. 6. Peso retenido acumulado en relación con el tamaño de partícula $(\Phi)$ de sedimentos en el punto de muestreo Paipote (PAI). En el recuadro interno del gráfico se muestran las líneas representativas de los tipos de transporte
En la figura 7 se expone la acumulación de sedimento para el punto de muestreo María Isabel. En otoño existió sólo transporte por suspensión, en tanto que en invierno se encontraron todos los tipos de transporte $(\mathrm{p}<0.05)$. En primavera hubo transporte por suspensión y una pequeña cantidad de sedimentos por tracción, mientras que en verano una gran cantidad de sedimento se transportó por saltación y una pequeña por suspensión.

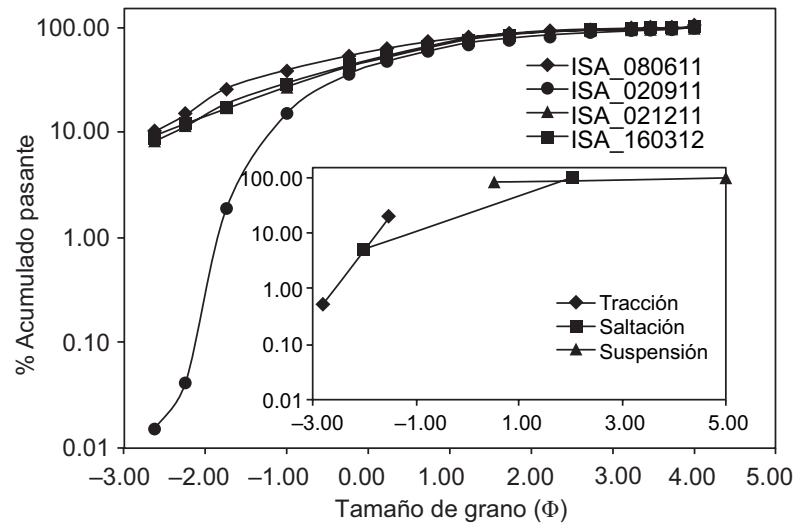

Fig. 7. Relación del peso retenido acumulado con el tamaño de partícula $(\Phi)$ de sedimentos en el punto de muestreo Fundo María Isabel (ISA). En el recuadro interno del gráfico se muestran las líneas representativas de los tipos de transporte

\section{Caracterización química}

La concentración de $\mathrm{Cu}$ fue alta y relativamente constante tanto en Paipote como en Cerrillos, si se compara con los otros puntos de muestreo (Fig. 8). En la dinámica de concentración se observó una importante disminución en invierno y un leve aumento en primavera. Las concentraciones de $\mathrm{Cu}$ son menores en los puntos Lautaro, Elisa de Bordos y María Isabel. En primavera y verano la concentración de $\mathrm{Cu}$ en Lautaro, María Isabel y Elisa de Bordos fue constante, pero siempre por arriba de las CEQG para el $\mathrm{Cu}(63 \mathrm{mg} / \mathrm{kg})$. Sin embargo, con el paso de los meses de otoño a invierno se observa una fuerte disminución de dicha concentración.

En general se observó que la concentración de $\mathrm{Pb}$ disminuyó en invierno, pero con una fuerte dispersión entre distintos puntos de muestreo (Fig. 9). En primavera se observa un aumento de la concentración, la cual se mantuvo sin mayor diferencia entre puntos de muestreo durante el verano. Destaca el fuerte aumento de la concentración en la transición 


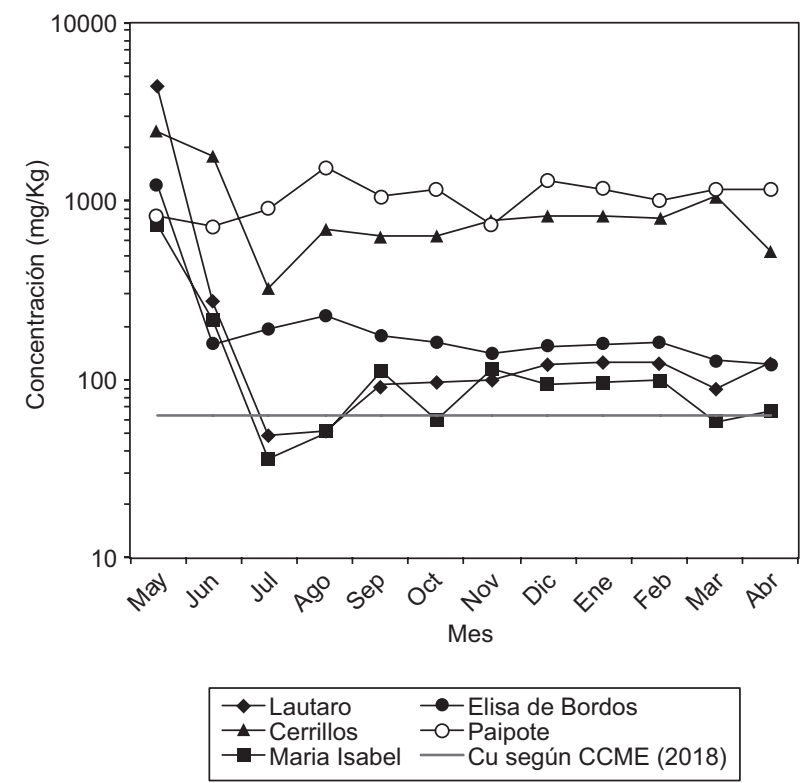

Fig. 8. Variación mensual de la concentración de Cu en los puntos de muestreo, de acuerdo con las normas canadienses de calidad ambiental (CCME 2018)

invierno-primavera en Elisa de Bordos. En general, la concentración se mantuvo por debajo las CEQG para el $\mathrm{Pb}(70 \mathrm{mg} / \mathrm{kg})$.

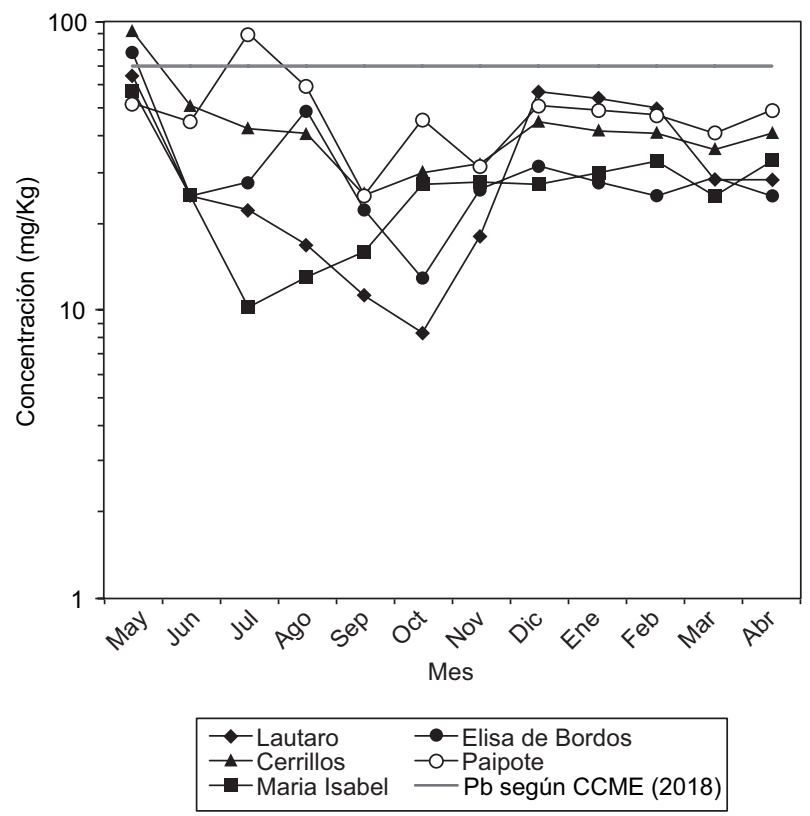

Fig. 9. Variación mensual de la concentración de $\mathrm{Pb}$ en los puntos de muestreo, de acuerdo con las normas canadienses de calidad ambiental (CCME 2018)
La concentración de As, en general, disminuyó en invierno y aumento en primavera-verano; en otoño hubo disminución paulatina, excepto para Paipote y Cerrillos, donde se mantuvo constante y elevada (Fig. 10). Todos los puntos presentaron generalmente concentraciones por arriba de las CEQG para el As $(12 \mathrm{mg} / \mathrm{kg})$.

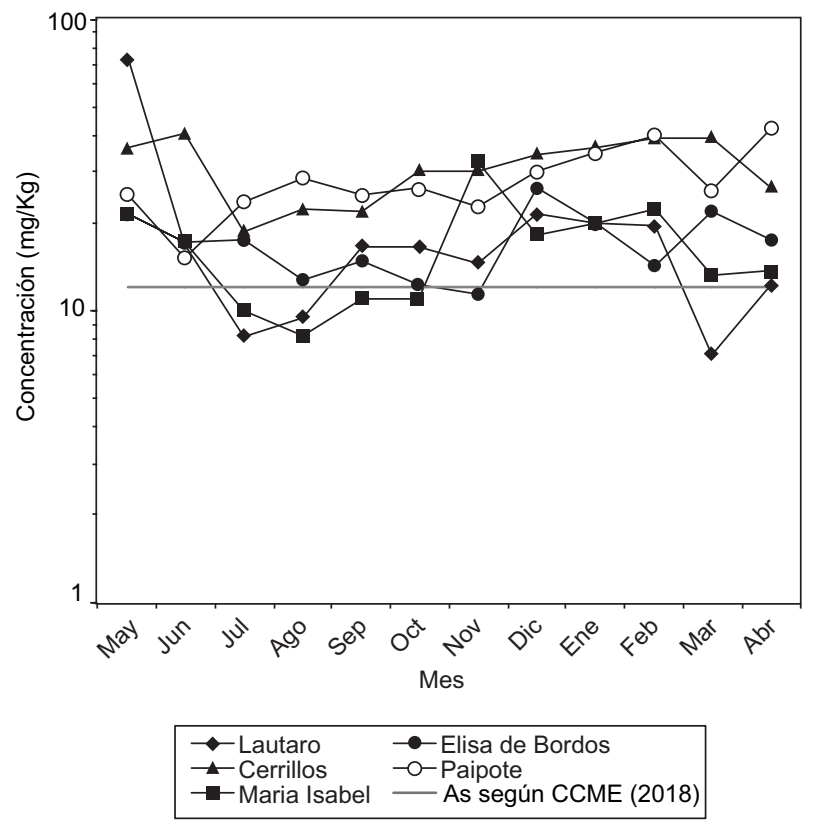

Fig. 10. Variación mensual de la concentración de As en los puntos de muestreo, de acuerdo con las normas canadienses de calidad ambiental (CCME 2018)

La concentración de $\mathrm{Hg}$ disminuyó en invierno, aumentó en primavera y disminuyó en verano-otoño en la mayoría de los puntos (Fig. 11). María Isabel fue el único punto que presentó máximos de concentración que sobrepasaron las CEQG, en tanto que los otros permanecieron con alta concentración y relativamente constantes. El límite permitido por las CEQG para el $\mathrm{Hg}$ es de $6.6 \mathrm{mg} / \mathrm{kg}$.

\section{DISCUSIÓN}

El río Copiapó está situado en la región de Atacama, zona árida de Chile. El río tiene su origen en la cordillera, en la confluencia de los ríos Jorquera y Pulido. Más abajo se junta con las aguas del río Manflas. El río cursa en sentido noroeste y tiene una longitud de $162 \mathrm{~km}$. Atraviesa las ciudades de Tierra 


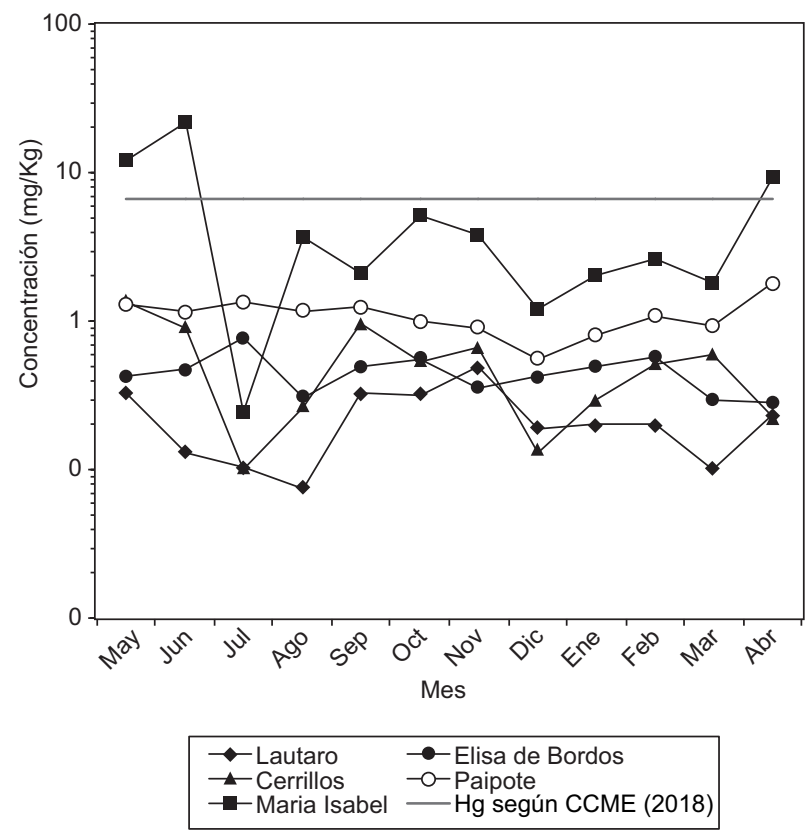

Fig. 11. Variación mensual de la concentración de $\mathrm{Hg}$ en los puntos de muestreo, de acuerdo con las normas canadienses de calidad ambiental (CCME 2018)

Amarilla y Copiapó, entre las cuales recibe la confluencia de la quebrada de Paipote, continuando en esa dirección hasta su desembocadura en el océano Pacífico. La cuenca presenta un relieve muy irregular y accidentado, predominando la alternancia de los valles transversales. El río tenía caudal antes de 1997, año en que un aluvión generó un gran caudal momentáneo; luego el río se secó en su segmento intermedio (incluyendo los puntos de muestreo Paipote y Cerrillos) por un periodo aproximado de 18 años. Por lo anterior, durante el muestreo entre 2011 y 2012 el río estaba seco casi en la totalidad de su trayecto alto y medio. No obstante, ha tenido importantes crecidas, como el aluvión de 2015. Desde ese año hasta el aluvión de 2017 el río tuvo un caudal muy variable. Actualmente presenta escorrentía variable con agua turbia, que se utiliza regularmente en el regadío de zonas agrícolas a pesar del contenido potencial de metales pesados y otros contaminantes. Los autores de este trabajo fueron testigos oculares de los cambios ocurridos en el río. De lo anterior se infiere que el escenario actual ha cambiado completamente, por lo que este trabajo es una importante líneade base de resultados de monitoreo anterior a los aluviones de 2015 y 2017, y no hay antecedentes de estudios anteriores o posteriores de monitoreo en periodos anuales.
Históricamente, las plantas mineras que usaba trapiches para la recuperación del Au mediante amalgamación con $\mathrm{Hg}$ (pequeña minería) se ubicaban en diferentes sitios cercanos al río entre los puntos de muestreo Elisa de Bordos y Paipote (sector de minería). Tal era la situación dominante hasta fines de la década de 1990, lo que dejó varios depósitos de relave abandonados. Por ejemplo, el relave llamado Totoralillo, que se encuentra al borde de la carretera entre los puntos de muestreo Elisa de Bordos y Cerrillos. No hay antecedentes completos ni publicados de la ubicación de la mayoría de los trapiches que existían en la región. El trapiche era un molino que utilizaba una rueda de cemento y acero accionada por un motor, la cual giraba en una solera abierta y producía la molienda del mineral, operando en húmedo (pulpa). La característica del diseño del trapiche permitía la recuperación del Au grueso en el mismo molino utilizando $\mathrm{Hg}$ para la amalgamación. La recuperación del Au fino, proveniente del rebalse del trapiche, se realizaba en un circuito de flotación que generaba un concentrado de $\mathrm{Au}$ y un relave o cola, que contenía los minerales no valiosos y se depositaba en sectores cercanos a la planta. Los relaves generados por el proceso presentaban tamaño granulométrico menor a $1 \mathrm{~mm}$ y contenían $\mathrm{Hg}$.

En los puntos Tranque Lautaro y Elisa de Bordos el río presenta una escorrentía superficial perenne, apreciándose un cambio en la dinámica de transferencia de los sedimentos en el tiempo debido a la deflación, las lluvias invernales de alta montaña y los deshielos de primavera-verano. El análisis granulométrico indica que durante los meses invernales el sedimento depositado se originó por transporte del tipo tracción, saltación y suspensión, en tanto que en primavera y verano los sedimentos depositados indican transporte sólo por tracción y saltación. Lo anterior indica que durante el invierno se produce sedimentación fina en este punto de la cuenca, probablemente asociada a una menor energía de caudal, mientras que en el resto del año la energía del caudal es suficiente para acarrear aguas abajo los sedimentos más finos.

En Puente Cerrillos y Paipote hay una leve alteración de la dinámica de transferencia de los sedimentos respecto al tiempo, la cual se relaciona con un río con caudal efímero. La granulometría de los sedimentos depositados indica que fueron trasportados por tracción, saltación y suspensión; pero es un lecho sin escorrentía permanente. En estos lechos, inmediatamente después de las lluvias, se acorta el campo de la saltación y se incrementa la presencia de sedimentos transportados por suspensión. Lo 
anterior se explica por la movilización de los sedimentos finos lejos del cauce por acción del viento, durante los meses en que el río permanece inactivo y seco, disminuyendo la presencia de sedimentos finos transportados por suspensión.

En el Fundo María Isabel no hubo cambio en la granulometría de los sedimentos en el tiempo. Lo anterior es esperable, ya que es una zona de pendientes bajas cercana a la costa, donde el río es alimentado por manantiales y canales que transportan agua ya usada en industrias y en la ciudad, y la lluvia es muy escasa. Por lo anterior, el transporte de sedimentos en esta zona es poco significativo, resultando un humedal costero con condiciones de baja energía de flujo.

La variación de la concentración de $\mathrm{Cu}, \mathrm{Pb}, \mathrm{Hg}$ y As en Tranque Lautaro y Elisa de Bordos puede explicarse por su situación de cauce con escorrentía superficial. La disminución de la concentración de los cuatro elementos durante el invierno podría explicarse por la inserción de sedimentos finos empobrecidos de estos elementos, provenientes de aguas arriba, los cuales diluyen la concentración. La mayor disminución de la concentración de los elementos en Tranque Lautaro con relación a Elisa de Bordos confirma que gran parte que los sedimentos finos transferidos desde la alta cordillera son represados en Tranque Lautaro y a Elisa de Bordos sólo llega un porcentaje marginal.

La alta concentración de $\mathrm{Cu}$ en Puente Cerrillos y Paipote, en relación con los demás puntos, podría deberse a la presencia de varias empresas de la minería de $\mathrm{Cu}$ en el sector. En particular, la concentración de $\mathrm{Cu}$ en la primera muestra de mayo de 2011 fue bastante alta en comparación con el resto, lo cual podría deberse a contaminación antrópica de la muestra entre la localidad de muestreo y el laboratorio. La concentración de $\mathrm{Cu}$ permaneció relativamente constante en el resto de los meses, sin embargo, las de $\mathrm{As} \mathrm{y} \mathrm{Pb}$ fueron variables, en concordancia con lluvias intermitentes en ese periodo que suman $30 \mathrm{~mm}$. Para ambos elementos, la concentración más baja en Puente Cerrillos en relación con Paipote puede estar relacionada con el lavado de los sedimentos y la dilución de las concentraciones al incorporarse sedimentos finos provenientes de aguas arriba. El aumento de la concentración en Paipote indicaría la transferencia de estos elementos desde una fuente próxima y también la presencia de relaves mineros en lugares próximos, concentración que podría haberse originado por escorrentía y/o deflación eólica. Respecto al $\mathrm{Hg}$, la concentración es constante en Paipote y disminuye hacia Puente
Cerrillos, lo cual podría explicarse como efecto de las lluvias, de forma similar a lo señalado para el descenso de la concentración de $\mathrm{Pb}$ y As en estos lugares.

En el punto Fundo María Isabel, la concentración de $\mathrm{Cu}, \mathrm{Pb}, \mathrm{Hg}$ y As varió estacionalmente entre uno y dos órdenes de magnitud; sin embargo, el análisis sedimentológico no indica variación importante en la dinámica de transferencia de sedimentos. La alta concentración de $\mathrm{Hg}$ puede ser explicada considerando la presencia aguas arriba de trapiches históricos utilizados en la molienda de Au. De igual manera, en las bahías de la comuna de Caldera (región de Atacama), se presentan problemas por la presencia de este elemento en los sedimentos marinos y en las cercanías a la costa (Valdés 2014).

La concentración de $\mathrm{Cu}$ y As estuvo siempre por arriba del límite permisible exigido por las CEQG, mientras que el $\mathrm{Pb}$ y el $\mathrm{Hg}$ mostraron, en general, concentración por debajo de la norma. La comparación de la concentración de los elementos con las CEQG abre el tema de importancia de la concentración relativa al fenómeno de bioacumulación.

El objetivo de catalogar la concentración de un contaminante como bajo o sobre la norma tiene una connotación puntual. Aunque la concentración de un elemento contaminante esté puntualmente bajo la norma, es propensa a incrementarse hasta sobrepasar los límites establecidos si se consideran los ciclos tróficos, debido a la acumulación del elemento en el tejido de los seres vivos. Como consecuencia de la bioacumulación, se propone el uso de especies de plantas acumuladoras como bioindicadores de contaminación (Benítez et al. 2011) en la gestión de planes ambientales urbanos (Alcalá et al. 2008), como agentes para tratamiento de suelos contaminados y como alternativa de fitorremediación (Arroyave et al. 2010, Madera-Parra et al. 2014). Por otra parte, los metales pesados pueden llegar a los ecosistemas acuáticos desde fuentes naturales y antrópicas, pudiendo bioacumularse en varios componentes de la cadena trófica; así, se cita la presencia de $\mathrm{Hg}$ en sedimentos y bivalvos, $\mathrm{Ag}$ y Se en hígado de salmónidos de los lagos Nahuel Huapi y Moreno (Argentina) (Rizzo et al. 2010), de modo que su impacto en el medio puede llegar a ser importante. Por lo anterior, la significación de la concentración de los elementos puede verse tanto desde el punto de vista del impacto geológico como del biológico.

Respecto al transporte de sedimentos en Tranque Lautaro, las lluvias invernales de alta montaña y los deshielos entre primavera y verano afectan la 
acumulación y el tipo de transporte. En Elisa de Bordos, este cambio en el transporte está directamente relacionado con la acumulación de sedimentos gruesos retenidos en Tranque Lautaro, ya que la escorrentía no presenta tanta energía y los sedimentos más finos son mayoritariamente transportados. Así, la variación en el modo de transporte de sedimentos está influida por cambios hidrológicos estacionales y la inclusión de sedimentos finos provenientes de aguas arriba, donde no hay fuerte intervención minera. Estos factores explicarían la fluctuación de la concentración a lo largo del año, en particular la disminución de la concentración de los elementos analizados en la transición otoño-invierno.

En el contexto de río efímero de los puntos Puente Cerrillos y Paipote, la acumulación de sedimento grueso y fino se ve afectada por deflación (transporte generado por el viento) y la escasez de lluvia. En este contexto, los sedimentos fluviales son trabajados nuevamente por la erosión del viento y cubiertos por depósitos eólicos. Lo anterior, junto a la fuerte presencia de la industria minera, determina altas y constantes concentraciones de elementos analizados, en relación con los puntos de muestreo situados aguas arriba. Un estudio de la concentración de estos elementos en la zona sería adecuado para conocer con mayor precisión la influencia de los procesos eólicos sobre la tasa de acumulación en el segmento de río efímero.

Finalmente, la acumulación y tipo de transporte de sedimentos en María Isabel no está afectada por las crecidas del caudal del río, ya que los sedimentos son movilizados desde manantiales provenientes de cerca de la costa, donde la lluvia es bastante escasa y no se presentan tributarios que aporten caudal. Las condiciones termodinámicas del agua dependen de las diferentes estaciones; lo que influiría en la concentración de los elementos (bioacumulación), por los organismos del humedal costero.

\section{CONCLUSIONES}

La variación temporal de la concentración de elementos en los sedimentos finos del río Copiapó, puede explicarse tanto por los cambios en la dinámica de transferencia de materia, como por la dinámica fluvial y eólica. Estos factores deberían considerarse en la planificación y manejo de los materiales desechados por la minería en un ambiente árido, en particular si se considera que el $\mathrm{Cu}$ y el As presentaron concentraciones superiores el límite permisible exigido por las CEQG.

\section{AGRADECIMIENTOS}

Este trabajo fue parte del proyecto Monitoreo de la Cuenca del río Copiapó (2011-2012), financiado por el Centro Regional de Investigación y Desarrollo Sustentable de Atacama, CRIDESAT, de la Universidad de Atacama, Copiapó, Chile.

\section{REFERENCIAS}

Alcalá J., Sosa M., Moreno M., Quintana C., Quintana G., Miranda S. y Rubio A. (2008). Metales pesados en vegetación arbórea como indicador de la calidad ambiental urbana: ciudad de Chihuahua. Multequina 17, 39-54.

Arroyave C., Araque P. y Peláez C.A. (2010). Evaluación de la bioacumulación y toxicidad de cadmio y mercurio en pasto llanero (Brachiaria dictyoneura). Vitae 17 (1), 45-49.

Benítez R., Calero V., Peña E. y Martin J. (2011). Evaluación de la cinética de la acumulación de cromo en el buchón de agua (Eichhornia crassipes). Rev. Bio. Agro. 9 (2), 66-73.

Bugueño M. (2014). Competencia de mecanismos bióticos y abióticos en el transporte de arsénico en el río Loa: efectos de macrófitas en los flujos y acumulación de arsénico. Tesis de Doctorado. Pontificia Universidad Católica de Chile, Santiago, Chile, 109 pp.

Castro C., Soto M., Fernández R., Märker M. y Rodolfi G. (2009). Impacto en la geodinámica actual del Valle de Nantoco, cuenca del río Copiapó, asociado a la reconversión productiva. Rev. Geogr. Norte Gd. 42, 81-99. DOI: $10.4067 / \mathrm{S} 0718-34022009000100006$

CCME (2018). Canadian Environmental Quality Guidelines. Canadian Council of Ministers of the Environment (en línea). http://ceqg-rcqe.ccme.ca/en/index. html.

Eberle W. (1998). Fortalecimiento del Departamento de Ingeniería y Gestión Ambiental de SERNAGEOMIN. El desarrollo histórico y los pasivos de la explotación minera en el curso medio y alto del Valle de Copiapó, III Región, Chile. SERNAGEOMIN, Santiago, Chile, $35 \mathrm{pp}$.

Espinoza F. (2013). Influencia de los factores naturales y antropogénicos en la geoquímica de sedimentos fluviales de las cuencas Vítor y San José de Azapa; XV región de Arica y Parinacota, Chile. Tesis de Licenciatura. Universidad de Chile, Santiago, Chile, 137 pp.

Folk R.L. y Ward W.C. (1957). Brazos River bar: A study in the significance of grain size parameters. J. Sediment Res. 27 (1), 3-26. DOI: 10.1306/74D70646-2B2111D7-8648000102C1865D 
Friedman G.M. y Sanders F.E. (1978). Principles of sedimentology. Wiley, Nueva York, EUA, 792 pp. DOI: $10.1002 /$ esp.3290040317

Jara C. (2007). Elementos base para la gestión ambiental del mercurio en Chile. Tesis de Licenciatura. Universidad de Chile, Santiago, Chile, 146 pp.

Madera-Parra C.A., Peña-Salamanca E.J. y Solarte-Soto J.A. (2014). Efecto de la concentración de metales pesados en la respuesta fisiológica y capacidad de acumulación de metales de tres especies vegetales tropicales empleadas en la fitorremediación de lixiviados provenientes de rellenos sanitarios. Ingeniería y Competitividad 16 (2), 179-188.

DOI: 10.25100/iyc.v16i2.3693

Rizzo A., Daga R., Arcagni M., Pérez S., Bubach D., Sánchez R., Ribeiro S. y Arribere M.A. (2010). Concentra- ciones de metales pesados en distintos compartimentos de lagos andinos de Patagonia Norte. Ecología Austral 20, 155-171.

Spalletti L. (1980). Paleoambientes sedimentarios en secuencias silicoclásticas. Publicaciones especiales de la Asociación Geológica Argentina, Serie B, Didáctica y Complementaria, número 8, Buenos Aires, Argentina, $175 \mathrm{pp}$.

Valdés J., Castillo A. (2014). Evaluación de la calidad ambiental de los sedimentos marinos en el sistema de Bahías de Caldera (27 $\left.{ }^{\circ} \mathrm{S}\right)$, Chile. Lat. Am. J. Aquat. Res. 42 (3), 497-513.

DOI: 103856/vol42-issue3-fulltext-10.

Visher G.S. (1969). Grain size distributions and depositional processes. J. Sediment Res. 39 (3), 10741106. DOI: 10.1306/74D71D9D-2B21-11D7$8648000102 \mathrm{C} 1865 \mathrm{D}$ 